\title{
CPAP promotes angiogenesis via interacting with and enhancing the activity of STAT3 in HCC
}

\author{
Liang-Yi Hung ${ }^{1}$, Ruo-Yu Chen ${ }^{1}$, Chia-Jui Yen ${ }^{1}$, Yao-Wen Liu², Yih-Jyh Lin ${ }^{1}$
}

${ }^{I}$ National Cheng Kung University, Taiwan, ${ }^{2}$ Kuo General Hospital, Taiwan

Hepatocellular carcinoma (HCC) is one of the malignant hypervascular tumors in which angiogenesis plays a vital role in tumor growth and metastasis. Our previous studies indicated that CPAP is overexpressed in HCC, and is positively correlated with recurrence and vascular invasion. In this study, we demonstrated that overexpressed CPAP increases tumor growth, angiogenesis, as well as metastasis ex vivo and in vivo. The expression level of CPAP has a positive correlation with activated STAT3 in HCC. Mechanistically, CPAP can act as a transcriptional co-activator of STAT3 to enhance IL-6-induced STAT3 activation by directly binding with STAT3. Overexpressed CPAP up-regulates several target gene expressions of STAT3 that are involved in angiogenesis, such as IL-8, CD44 and MCAM. The expression of CPAP mRNA is positively correlated with the levels of IL-8, CD44 and MCAM mRNAs in HCC. Importantly, IL -6/STAT3-mediated angiogenesis is significantly increased by overexpressed CPAP, and can be blocked by decreased expression of IL-8. Taken together, our results suggest that overexpressed CPAP interacts with STAT3 to enhance its activity and therefore leads to angiogenesis. Our findings shed light on the importance of CPAP to act as a potential therapeutic target in inhibiting the angiogenesis pathway. 